\title{
ARE NOVAE STANDARD CANDLES?
}

\author{
SHLOMI PISTINNER ${ }^{1}$, GIORA SHAVIV ${ }^{2}$, \\ PETER H. HAUSCHILDT ${ }^{3}$, SUMNER STARRFIELD ${ }^{3}$ \\ 1. Department of Physics, Ben-Gurion University, \\ Beer Sheba 84105, Israel \\ 2. Department of Physics, Technion, Haifa 32000, Israel \\ 3. Department of Physics \& Astronomy, Arizona State \\ University, Tempe, AZ 85287-1504, USA
}

\section{Introduction}

Novae in outburst can be used as distance indicators. The distance measurement process is considerably simplified if novae could be regarded as standard candles. The term standard candles in the context of this paper means that the absolute magnitude at maximum for different novae is, to a good approximation, constant. The $t_{3}-M_{B}$ diagram (Schmidet 1957) implies that this is the case for M31 and LMC novae (Della Valle \& Livio 1995). However, this conclusion does not necessarily apply to non Local Group novae (Livio 1992; Della Valle \& Livio 1995); for example the Virgo novae are not similar to Local Group novae (Della Valle \& Livio 1995). Here we focus on a question of principle, namely: Does the fact that novae have similar spectra imply that they have similar luminosities?

Our discussion is limited to the very early phase in which a pseudophotosphere forms within the ejecta, the so-called photospheric phase. Classical methods of stellar atmosphere analysis cannot be applied directly to nova atmospheres in this stage, because they are expanding and because of their large spatial extent. Hauschildt et al. $(1992,1994,1995)$ have devised methods to simulate the expanding atmospheres of novae in this phase and thereby, obtained elemental abundances, effective temperatures, and atmospheric structures.

The detailed physics of a nova model atmospheres has been recently discussed by Hauschildt et al. (1995). The synthetic spectra they have calculated indicate that, over certain wavelength ranges, in which continuum processes dominate line processes, the spectra are relatively insensitive to 
the input parameters. In a recent work Pistinner et al. (1995) explored the sensitivity of early nova spectra to the input parameters. They identify spectral regions in which the continuum spectra are insensitive to the input parameters and certain wavebands in which they are sensitive to the input parameters. Pistinner et al. (1995) have identified the dilution factor as a small number, using it in a small parameter expansion they were able to find an analytical solution to the radiative transfer equation and provide predictions. These predictions account for the insensitivity of the spectra to the input parameters.

Nova atmospheres differ from normal stellar atmospheres by their motion. However, another important fact is frequently overlooked. Namely, nova atmospheres differ by being dominated by scattering and not by absorption in the continuum. The purpose of this paper is to study the effects resulting from this fact.

\section{Continuum similarities of nova atmospheres}

For the sake of brevity the element of motion will not be included in this presentation. The element of motion can be rigorously accounted for (Pistinner et al. 1995). Consider the static configuration shown in Fig. 1. Three distinct zones are marked: (a) the 'photosphere' (marked in Fig. 1 as ZA) where $\tau_{\text {ext }}=\tau_{\text {scat }}+\tau_{\text {abs }} \leq 1$ and $\tau_{\text {scat }} \gg \tau_{\text {abs }}$, where $\tau_{\text {ext }}, \tau_{\text {scat }}, \tau_{\text {abs }}$ are the optical depth for extinction, scattering and absorption in the continuum respectively; (b) a region (marked $\mathrm{ZB}$ ) where $\tau_{\text {scat }} \geq \tau_{\text {abs }}$; and (c) a region (marked $\mathrm{ZC}$ ) where absorption dominates. This particular configuration leads to the 'continuum similarity' (CS) of synthetic novae spectra over large range of wavelengths. By CS we mean that the continuum spectra are insensitive to the input parameters of the model atmospheres used to calculate the spectra. Specifically, significant changes to the input parameters do not result in significant changes to the emergent spectra. Another way to define CS is by noting that there are circumstances where different combinations of input parameters yield practically identical spectra.

An example of similar models is given in Table 1. In particular the spectra are insensitive to the luminosity (in the above case the luminosities change by a factor of 10 !), implying that high similarities between the observed spectra of different novae cannot be used to argue that all nova have similar luminosities.

Therefore, any argument about novae being standard candles based on high spectral similarities is patently invalid. This does not imply that novae in the Local Group are not approximately standard candles. Other techniques, which involve the temporal changes in the brightness of the nova, may indeed show that these novae have comparable luminosities. However, 


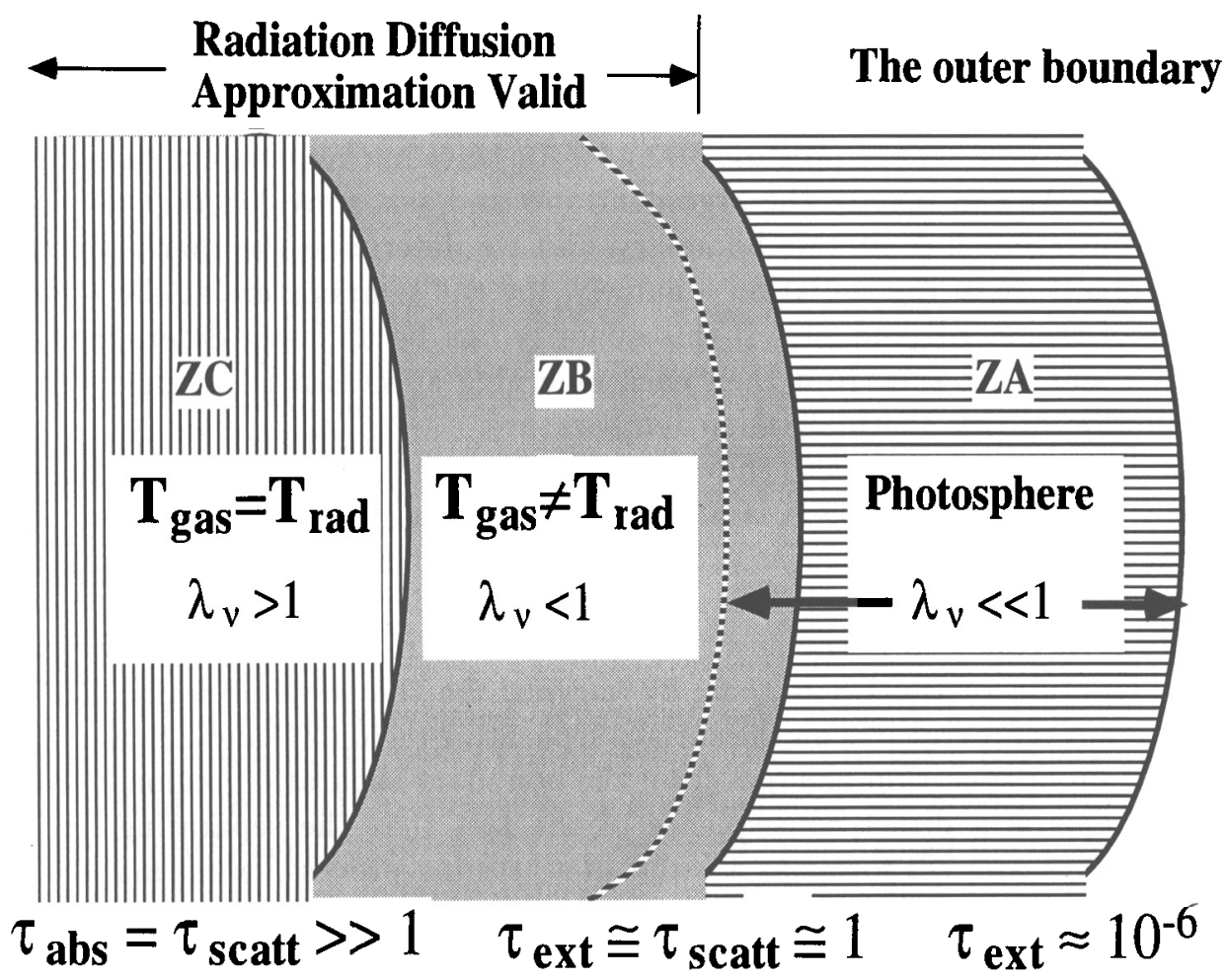

Figure 1. Different zones in a nova atmosphere.

TABLE 1. Models

\begin{tabular}{lc}
\hline$T_{\text {eff }}$ & $R_{\text {phot }}$ \\
\hline $15000 \mathrm{~K}$ & $4.610^{12} \mathrm{~cm}$ \\
$18000 \mathrm{~K}$ & $1.010^{12} \mathrm{~cm}$ \\
\hline
\end{tabular}

the extrapolation of these relations to other galaxies has been shown to yield larger scatter than for the Local Group (Della Valle \& Livio 1995).

To understand the underlying physical reason for the similarity effect consider first region ZA. In this region both the lines and the continua are nearly purely scattering due to the low density of the envelope. This is the well known Schuster (1905) problem. It can be shown that this region has rather small imprints of the continuum and the lines. 
The continuum formation region is region $\mathrm{ZB}$, in this region the memory of the blackbody radiation emerging from region $\mathrm{ZC}$ is processed. A synthetic spectrum calculation is performed to predict the emergent flux at the boundary between region $\mathrm{ZB}$ and $\mathrm{ZA}$ ( since in this case ZA has a small effect). We can reverse the argument now and ask, provided that we know the emergent flux at this boundary, can we determine the thermalization temperature of the radiation - namely, the gas temperature at the boundary of region ZB and ZC? If this quantity can be uniquely determined for each emerging spectrum then each emerging spectrum is a single valued function of this thermalization temperature.

Pistinner et al. (1995) show that in this case the only physical quantity that governs the problem is a quantity known as the dilution factor. This quantity can be written as a combination of input parameters. Thus, the thermalization temperature is not a unique function of the input parameters and as a result so is the spectra. This is the cause of the CS. A heuristic explanation of the $\mathrm{CS}$ is that, by varying the thickness of region $\mathrm{ZB}$ for different temperatures on the surface separating region $\mathrm{ZC}$ and $\mathrm{ZB}$, a similar continuum spectrum emerges from the boundary separation region ZA and $\mathrm{ZB}$. This is a direct consequence of the fact that the separating line of region $\mathrm{ZA}$ and $\mathrm{ZB}$ forms as a result of scattering process. In normal stellar atmospheres region $\mathrm{ZB}$ does not exist!

\section{Conclusion}

Similar spectroscopic appearance of observed novae can not be used to argue that they have similar luminosities and thus are standard candles. The element abundance and the luminosity can be determined only to within a given transformation. The UV spectral range is not susceptible to spectral similarities and thus, high quality UV observations might aid in removing the above discussed ambiguity.

\section{References}

Della Valle, M., Livio, M., 1995, Ap. J., 452, 704

Hauschildt, P.H., Wehrse, R., Starrfield, S., Shaviv, G., 1992, Ap. J., 393, 307

Hauschildt, P.H., Starrfield, S., Austin, S., et al,, 1994, Ap. J., 422, 831

Hauschildt, P.H., Starrfield, S., Shore, M., et al., 1995, Ap. J., 447, 829

Livio, M., 1992, Ap. J., 393, 516

Pistinner, S., Shaviv, G., Hauschildt, P.H., Starrfield, S., 1995, Ap. J., 451, 724

Schmidet, Th., 1957, Z. Astrophys., 41, 182

Schuster, A., 1905, Ap. J., 21, 1 